\title{
Wireless Holographic Image Communications Relying on Unequal Error Protected Bitplanes
}

\author{
Yongkai Huo, Péter Tamás Kovács, Student Member, IEEE, Thomas J. Naughton, and Lajos Hanzo, Fellow, IEEE
}

\begin{abstract}
Holography is considered to be one of the most promising techniques of goggle-free visualization for the nearfuture. We consider wireless transmission of digital holograms, which are partitioned into multiple bitplanes that are then independently encoded by a forward error correction (FEC) code for transmission over wireless channels. The coding rates of these bitplanes will be optimized at the transmitter for the sake of achieving an improved holographic peak signal-to-noise ratio (PSNR) at the receiver. Our simulation results show that up to $2.6 \mathrm{~dB}$ of $E_{b} / N_{0}$ or $12.5 \mathrm{~dB}$ of PSNR improvements may be achieved when employing a recursive systematic convolutional code.
\end{abstract}

Index Terms-Holography, unequal error protection, wireless video transmission.

\section{INTRODUCTION}

$\mathbf{H}$ OLOGRAPHY has been widely researched since its invention by Gabor [1]. We commence by introducing the holography concept, followed by the current state-of-the-art in its compression and transmission. We continue by outlining the motivation and focus of our paper and present its structure.

\section{A. Holography}

Holography [1] constitutes a sophisticated technique of recording and reconstructing both the amplitude and phase of an optical wavefront relying on the interference and diffraction imposed by an object on visible light. Holography ${ }^{1}$ [1], including optical holography, computer generated holography (CGH), and digital holography $(\mathrm{DH})$ are being actively researched at the time of writing [2]-[6]. In [4], CGHs were generated using a small number of multiview images captured by appropriately arranged cameras. An efficient generation of the CGH was

Manuscript received February 6, 2016; revised December 30, 2016; accepted January 6, 2017. Date of publication January 20, 2017; date of current version August 11, 2017. This was work was supported by the European Research Council's Advance Fellow Grant Beam-Me-Up and by the Royal Society's Wolfson Research Merit Award.

Y. Huo is with the College of Computer Science and Software Engineering, Shenzhen University, Shenzhen 518060, China (e-mail: ykhuo@szu.edu.cn or yh3g09@ecs.soton.ac.uk).

P. T. Kovács is with the Holografika Kft, Budapest 1192, Hungary (e-mail: p.kovacs@holografika.com).

T. J. Naughton is with the Department of Computer Science, National University of Ireland, Maynooth W23, Ireland (e-mail: tomn@cs.nuim.ie).

L. Hanzo is with the School of Electronics and Computer Science, University of Southampton, Southampton SO17 1BJ, U.K. (e-mail: 1h@ecs.soton.ac.uk).

Color versions of one or more of the figures in this paper are available online at http://ieeexplore.iee.org.

Digital Object Identifier 10.1109/TVT.2017.2656798

${ }^{1}$ The word "holography" originated from the Greek words "holos" meaning whole or entire and "graphein" meaning to write. proposed in [6]. The European Real 3-D research project [2], [7] aimed for capturing both 3-D and 4-D real-world objects, as well as for the processing and display of $\mathrm{DH}$.

1) Optical Holography: Optical holography allows us the holographic images to be recorded and reconstructed using a white-light illumination source [8] or a illuminating laser [9]. According to the reconstruction method, holograms may be classified as reflection [8] and transmission holograms [9]. In this treatise, we focus on the transmission holograms, which may be recorded and reconstructed as in Fig. 2. Optical holography entails the family of techniques that record a hologram using traditional acetate-based film and then reconstructs the image optically using an illumination light.

2) Computer Generated Holography: CGH was first proposed by Brown and Lohmann [10], Lohmann and Paris [11], which allows us to generate holograms with the aid of sophisticated mathematical manipulations of an object that does not physically exist but can be described in mathematical terms. Hence, CGH refers to the family of techniques that generate a hologram from virtual objects using mathematical manipulations and then reconstruct the image optically using classic laser illumination methods [9].

3) Digital Holography: Goodman and Lawrence [12] proposed the principle of DH [13], which yields images reconstructed with the aid of computations from a digitized Fourier hologram that was electronically detected by a vidicon camera [12] from an optically recorded hologram. Based on this principle, the fundamental theory of $\mathrm{DH}$ was conceived by Yaroslavskii and Merzlyakov [14]. In a nutshell, DH refers to the class of techniques that record a hologram digitally and reconstruct the image using numerical manipulations.

\section{B. Compression and Transmission}

Holography has been widely researched for diverse applications [15], [16], such as deformation analysis [17], communications [18], microscopy [13], etc. Since digital holograms, including the $\mathrm{CGH}$ and DH holograms, are stored in digital form, suitable compression and transmission techniques have to be investigated for the sake of reducing the storage required in a hard-drive for example, or the transmission bandwidth and the transmission power required for distributing the holograms [19].

A number of compression techniques were discussed in [20] and [21], including classic lossless compression, quantization, Fourier-domain processing, wavelet analysis, etc. The lossy 


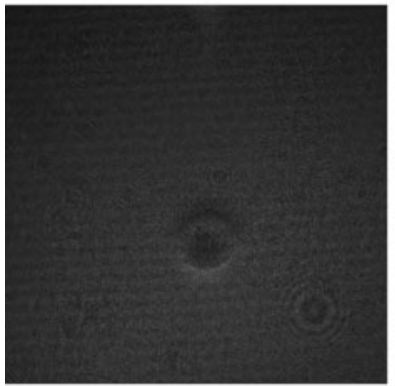

(a)

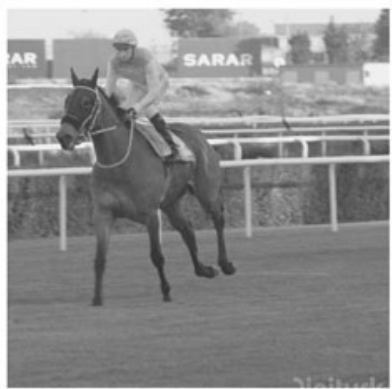

(b)

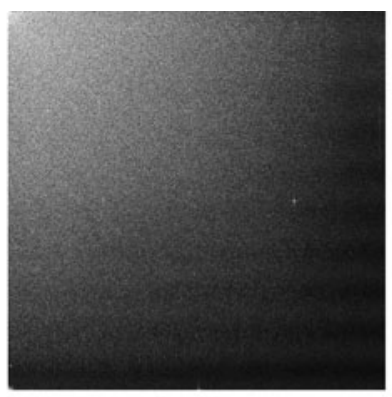

(c)

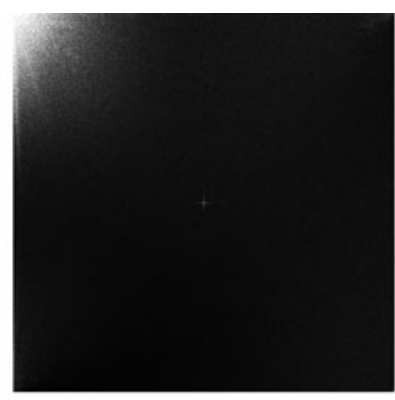

(d)
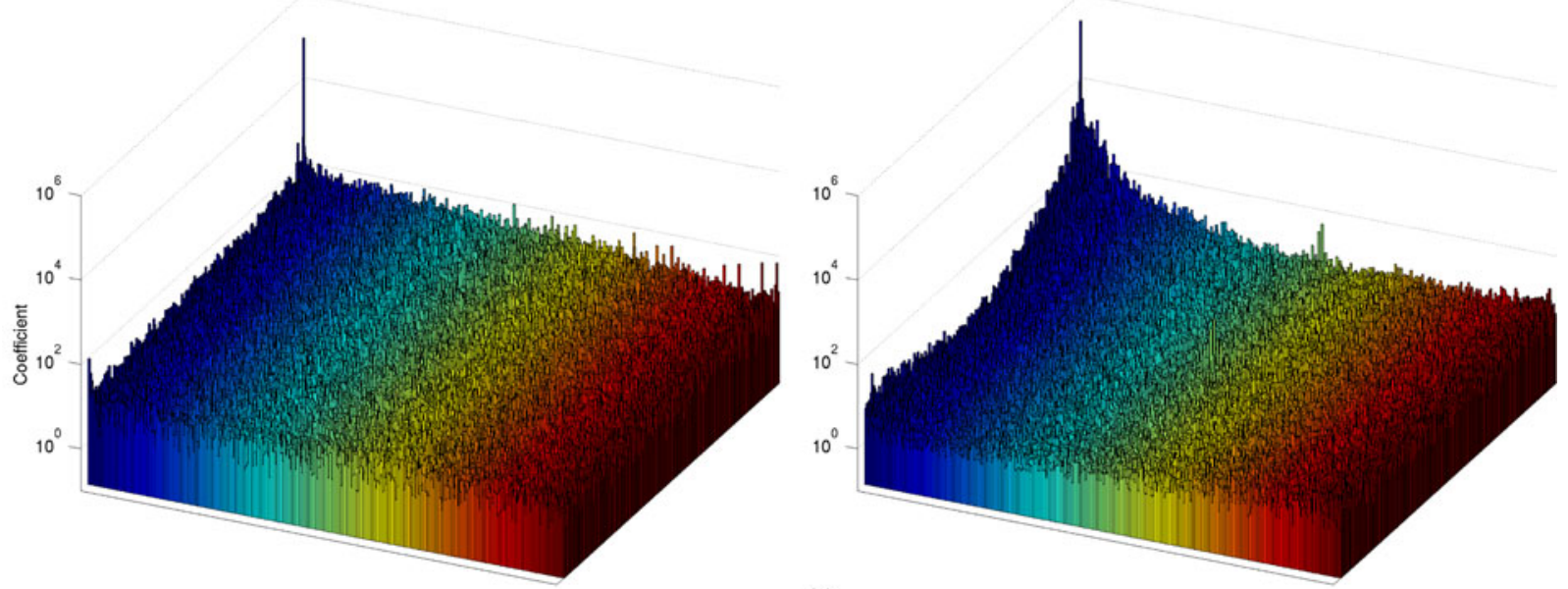

(e)

Fig. 1. Comparison of the hologram of a Coil to a jockey image. (a) Hologram of a Coil. (b) Jockey image. (c) DCT domain, Coil hologram. (d) DCT domain, jockey image. (e) DCT Coefficients, Coil hologram versus jockey image.

compression of phase-shift-based digital holograms was investigated in [22], where both the real and imaginary streams were quantized, followed by a bit-packing operation. The wavelet-like basis functions, namely the so-called Fresnelets were investigated in [23] and [24]. Wavelet analysis was employed in [25] for the compression of complex-valued digital holograms of 3-D real-world objects, where the thresholding and quantization of the wavelet coefficients was invoked, followed by the lossless encoding of the quantized data. In [26], the wavelet-bandelets transform was employed for hologram compression. The widely known scalable video coding method of [27] was employed in [28] for compressing holographic video.

However, there is a paucity of literature on the transmission of digital holograms. A wireless holographic video transmission system was proposed in [29], where the holograms were transformed into a bitstream, and then transmitted over both wireless LAN and Bluetooth. In [30], the authors investigated the transmission of holograms through a multimode optical fiber by shaping the wavefront of the input beam with the aid of a spatial light modulator. Transmission of holograms and 3-D image reconstruction using white LED light was investigated in [31]. The authors of [32] proposed a method to transmit CGH using an infrared-rays, where the hologram was compressed before transmission.

\section{Our Motivation}

The distribution of digital hologram pixels is rather different from that of traditional photographic image pixels [24], [33], [34], as exemplified in Fig. 1 portraying the hologram of a simple Coil and a jockey image. The visual comparison of a hologram and of a traditional image is shown by Fig. 1(a) and (b), whereas corresponding discrete cosine transform (DCT) coefficients are compared in Fig. 1(c)-(e). As displayed in Fig. 1(e), high valued DCT coefficients of the correlated jockey image tend to be in the top left corner associated with the low-frequency components, which indicates that a compressed version of the jockey image may be represented by a small faction of the coefficients, thereby achieving high compression. In contrast to the jockey image, the DCT coefficients of the Coil hologram tend to be more uniformly scattered over the whole DCT coefficient plane. Hence, the traditional image compression techniques, such as the joint photographic experts group's schemes [35] and the intraframe compression mode of H.264/AVC [36] are inefficient for digital uncorrelated holograms.

\section{Our Focus}

As discussed above, digital holograms may be widely utilized in future applications. However, apart from [30], the 


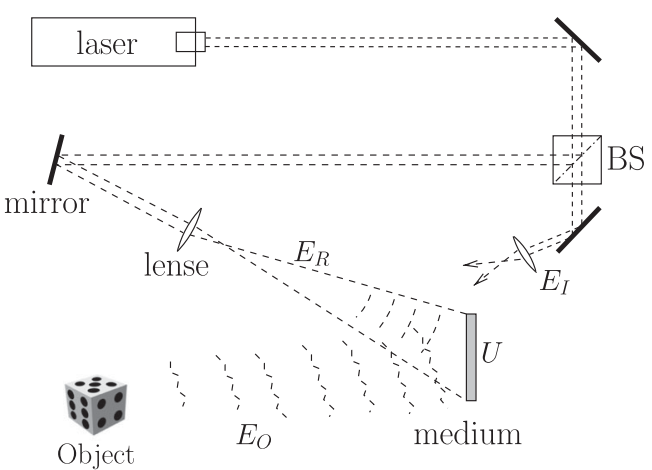

(a)

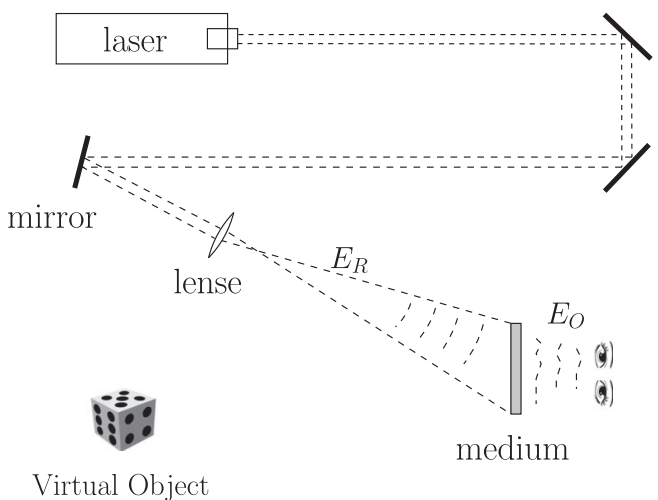

(b)

Fig. 2. Optical set-up of holography. (a) Recording. (b) Optical reconstruction.

transmission of digital holograms has rarely been researched. Hence, we embark on tackling this open problem by investigating the transmission of digital holograms through wireless channels. Furthermore, since no widely acclaimed compression algorithms have been developed in the open literature, we directly transmit uncompressed holograms with the objective of reconstructing the original high-quality decoded digital holograms at the receiver. Explicitly, we propose an optimized unequal error protection (UEP)-based forward error correction (Opt-UEP-FEC) coded system, where the holograms will be transmitted bitplane by bitplane after FEC. We will optimize UEP [37] rates of the bitplanes for the sake of maximizing the quality of the received digital holograms. Note that our previous work [37]-[39] optimized the coding rates of the different layers in scalable video, where the less important layers rely on the more important layers for their decoding. By contrast, in this contribution, we optimize the coding rates of different bitplanes, which are independent of each other for decoding.

Hence, the novelty of this paper is listed as follows.

1) We study the transmission of uncompressed holograms based on unequal error protected bitplanes.

2) We optimize the coding rates of unequal FEC protection. Our solution may be applied to arbitrary channels, modulation arrangements and to noniterative FEC schemes.

3) Substantial system performance improvements have been achieved compared to conventional equal error protection (EEP) schemes.
TABLE I

SyMBOL DEFINITION, WHERE $0 \leq i<m$ INDICATES THE BITPLANE INDEX

\begin{tabular}{ll}
\hline \hline Symbol & \\
\hline$U$ & The original hologram, as displayed in Fig. 3 \\
$m$ & Number of bits/pixel for the hologram $U$ \\
$u_{i}$ & The $i$ th bitplane of the hologram $U$ \\
$\bar{u}_{i}$ & The bit sequence linearly indexed ${ }^{2}$ The bits of a $2 \mathrm{D}$ bitplane are \\
& indexed using a one-dimensional formulation. \\
& from the bitplane $u_{i}$ \\
$r_{i}$ & FEC coding rate of the bitplane $u_{i}$ \\
$x_{i}$ & The FEC encoded sequence of the bitplane $u_{i}$ \\
$y_{i}$ & The received version of sequence $x_{i}$ \\
$\hat{\bar{u}}_{i}$ & The decoded version of bit sequence $\bar{u}_{i}$ \\
$\hat{u}_{i}$ & The decoded version of bit sequence $u_{i}$ \\
$\hat{U}$ & The reconstructed hologram at the receiver \\
\hline \hline
\end{tabular}

The rest of the paper is organized as follows. Section II will briefly introduce the basic principles of both optical holography, as well as of CGH and DH. Then the architecture of the proposed system will be presented in Section III, followed by the proposed coding rate optimization in Section IV. Then the system's performance will be characterized in Section V. Finally, Section VI concludes the paper.

\section{BASICS OF HOLOGRAPHY}

\section{A. Recording}

The optical set-up of hologram recording is illustrated in Fig. 2(a), where an object, a coherent light source, such as a laser, as well as mirrors, lenses, and a recording medium are employed. The laser is split into a pair of partial waves by the beam splitter (BS), namely the waves $E_{I}$ and $E_{R}$ of Fig. 2(a). The wave $E_{I}$ of Fig. 2(a), which is referred to as the illumination wave, illuminates the object and it is scattered by the object's surface. The scattered wave, which is also referred to as the object wave, $E_{O}$ is then reflected onto the recording medium $U$ of Fig. 2(a). The wave $E_{R}$, which is also referred to as the reference wave, illuminates the recording medium directly. Finally, the interference pattern created by this pair of waves will be recorded by the medium $U$, which is the resultant hologram. A conventional photographic plate may be employed as the recording medium of Fig. 2(a) for optical hologram recording. By contrast, a CCD may be invoked for digital hologram recording.

We assume that the complex-valued amplitude of the object wave $E_{O}$ of Fig. 2(a) is described by

$$
E_{O}(w, h)=a_{O}(w, h) \cdot \exp \left[i \varphi_{O}(w, h)\right]
$$

where the real-valued amplitude is $a_{o}$ and the phase is denoted by $\varphi_{o}$. The complex-valued amplitude of the reference wave $E_{R}$ of Fig. 2(a) is described by

$$
E_{R}(w, h)=a_{R}(w, h) \cdot \exp \left[i \varphi_{R}(w, h)\right]
$$

where the real-valued amplitude is denoted by $a_{R}$ and the phase by $\varphi_{R}$. Then, the intensity of the interference pattern of the two waves at the surface of the recording medium $U$ of Fig. 2(a) can 

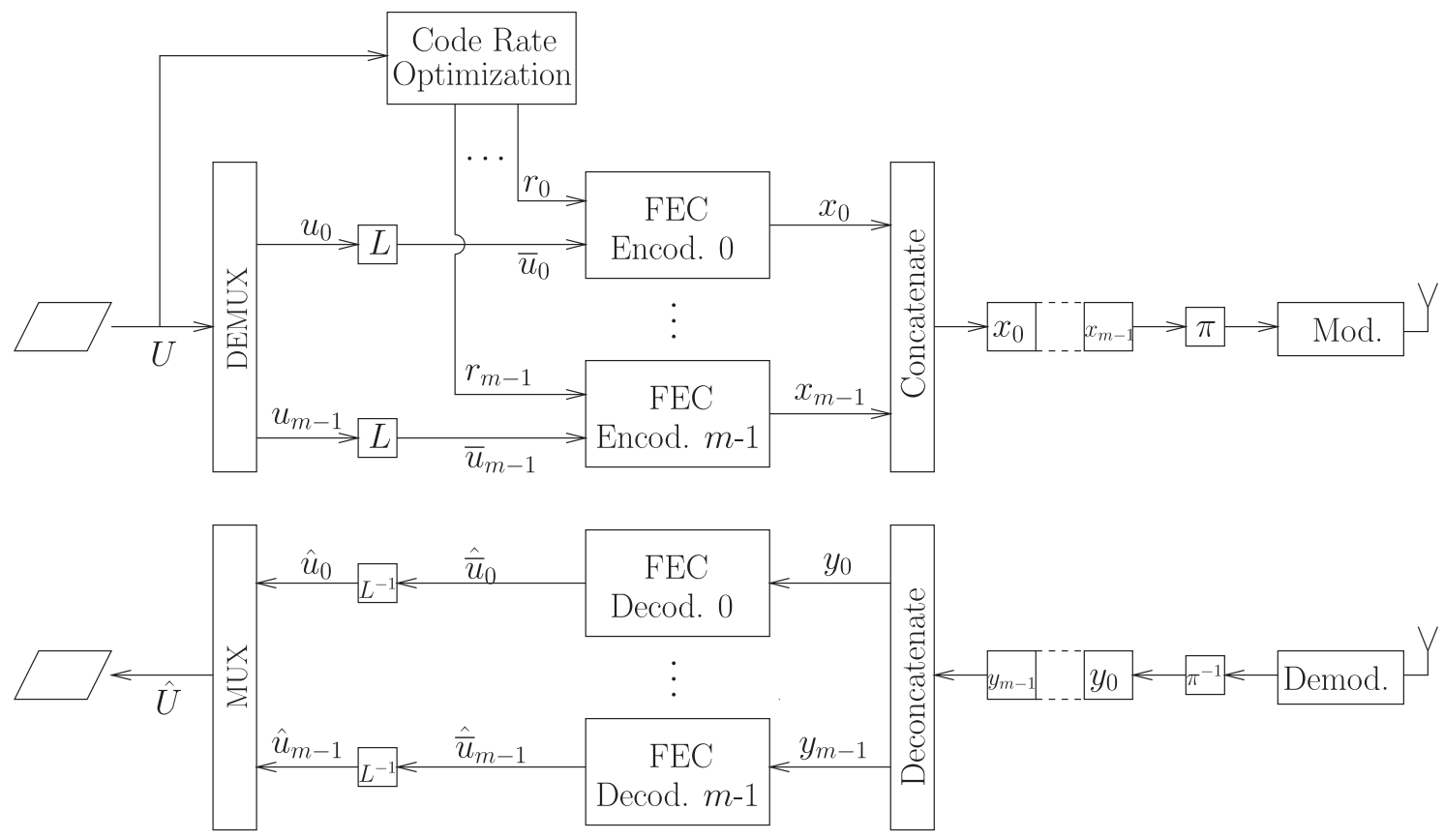

Fig. 3. Block diagram of the proposed Opt-UEP-FEC system, where $m$ is the bit-depth of the hologram, while $r_{0}, \ldots, r_{m-1}$ represent the code rates of the FEC encoders $0, \ldots, m-1$, respectively. The "code rate optimization" block will be detailed in Section IV.

be expressed as

$$
U(w, h)=\left|E_{O}(w, h)+E_{R}(w, h)\right|^{2}
$$

For $\mathrm{CGH}$, the hologram is created by calculating (1) to (3), where the mathematical model of the object is known. More details about the CGH may be found in [40] and [41].

\section{B. Reconstruction}

The optical reconstruction set-up is illustrated in Fig. 2(b), where a coherent laser light source, mirrors, lenses, and a hologram are employed. The reference wave $E_{R}$ illuminates the hologram $U$, which results in a virtual image, that may be viewed by the observer.

The amplitude transmittance $H(w, h)$ of the recording medium is proportional to the intensity $U(x, y)$ of the hologram, which may be expressed as

$$
H(w, h)=H_{0}+\beta \tau \cdot U(w, h)
$$

where $\beta$ represents the slope of the amplitude transmittance versus the exposure characteristic of the light sensitive material, whereas $\tau$ is the exposure time, and $H_{0}$ is the amplitude representing the unexposed plate [15]. The associated hologram reconstruction can be described mathematically as the product of the amplitude transmittance $H(w, h)$ and the reference wave $E_{R}$ of Fig. 2(b), namely $E_{R}(w, h) H(w, h)$.

For $\mathrm{CGH}$, the digital hologram is first printed on film, which will then be optically reconstructed. For $\mathrm{DH}$, the hologram will be numerically reconstructed by simulating the optical reconstruction process [15], [40], [41].

\section{SySTEM ARCHITECTURE}

In this section, we introduce the proposed UEP-based FEC coded (Opt-UEP-FEC) system conceived for holographic communications, whose system model is detailed in Fig. 3. We focus on the general architecture of the transmitter and receiver, whereas the "code rate optimization" block will be detailed in Section IV. Let us commence by defining the notations as in Table I.

\section{A. Transmitter Model}

At the transmitter of Fig. 3, the original hologram $U$ is demultiplexed into the classic bitplanes $u_{0}, \ldots, u_{m-1}$ by the $D E$ $M U X$ block, where $u_{0} / u_{m-1}$ represents the most/least significant bitplane. ${ }^{3}$ Meanwhile, the original hologram $U$ is input to the "code rate optimization" block, which will generate the optimized coding rates $r_{0}, \ldots, r_{m-1}$ for the bitplanes $u_{0}, \ldots, u_{m-1}$, respectively. Afterwards, each bitplane $u_{i}(0 \leq i<m)$ is encoded as follows.

1) The bitplane $u_{i}$ will be linearly indexed to generate the sequence $\bar{u}_{i}$ by the block $L$.

2) The resultant sequence $\bar{u}_{i}$ is then encoded by the FEC encoder $i$, which generates the encoded bit sequence $x_{i}$.

Finally, the bit sequences $x_{0}, \ldots, x_{m-1}$ are concatenated into a joint bitstream for transmission. The interleaver $\pi$ of Fig. 3 is employed for interleaving the joint bitstream before the modulation and transmission over nondispersive uncorrelated Rayleigh fading wireless channels. Although we will employ a simple binary phase-shift keying (BPSK) modulator in the "Mod." block, arbitrary transceivers may be applied in our proposed system.

\footnotetext{
${ }^{3}$ Assume a 2-D image has $m$-bits/pixel, where each pixel may be split into $m$ bits. All the bits having the same significance are collected in a bitplane.
} 
TABLE II

SYMBOL DEFINITION, WHERE $0 \leq i<m, 0 \leq j<m$ INDICATE THE BITPLANE INDEX

\begin{tabular}{|c|c|}
\hline Symbol & Definition \\
\hline$W$ & The width of the hologram \\
\hline$H$ & The height of the hologram \\
\hline$R$ & Overall coding rate of the system \\
\hline$U(\rho)$ & $\begin{array}{l}\text { The pixel at position } \rho=(w, h) \text { of the hologram } U \text {, namely } \\
U(w, h)\end{array}$ \\
\hline$\hat{U}(\rho)$ & $\begin{array}{l}\text { The pixel at position }(w, h) \text { of the received and reconstructed } \\
\text { hologram }\end{array}$ \\
\hline$u_{i}(\rho)$ & $\begin{array}{l}\text { The } i \text { th bit of the pixel } U(\rho) \text {, namely the bit at position }(w, h) \text { of the } \\
\text { bitplane } u_{i}\end{array}$ \\
\hline$\hat{u}_{j}(\rho)$ & The $j$ th bit of the pixel $\hat{U}(\rho)$ \\
\hline$p\left[u_{i}(\rho)=1\right]$ & Indicates the probability that the bit $u_{i}(\rho)$ is 1 \\
\hline$p\left[\hat{u}_{j}(\rho)=1\right]$ & Indicates the probability that the bit $\hat{u}_{j}(\rho)$ is 1 \\
\hline
\end{tabular}

\section{B. Receiver Model}

At the receiver, BPSK demodulation, deinterleaving, and deconcatenation are performed, as seen in Fig. 3, which generate the soft information $y_{0}, \ldots, y_{m-1}$ for the bitplanes $u_{0}, \ldots, u_{m-1}$, respectively. Then, each bitplane $u_{i}(0 \leq i<m)$ is estimated as follows.

1) The soft information $y_{i}$ is decoded by the FEC decoder $i$ generating the bit sequence $\hat{\bar{u}}_{i}$, which is the estimated version of bit sequence $\bar{u}_{i}$.

2) The sequence $\hat{\bar{u}}_{i}$ will then be reformatted to the bitplane $\hat{u}_{i}$ by the the block $L^{-1}$, where $\hat{u}_{i}$ is the estimated version of the bitplane $u_{i}$.

Finally, the estimated bitplanes $\hat{u}_{0}, \ldots, \hat{u}_{m-1}$ are reconstructed into the final estimated hologram $\hat{U}$ by the "MUX" block.

\section{CODING RATE OPTIMIZATION}

In this section, we detail the "code rate optimization" block of Fig. 3. This "code rate optimization" block has the task of finding the specific FEC coding rates $r_{0}, \ldots, r_{m-1}$ required for encoding the different-significance bitplanes $u_{0}, \ldots, u_{m-1}$. We denote the position of a specific pixel by $\rho=(w, h)$ in the intensity hologram frame for notational simplicity. Note that real valued numbers are utilized for representing a pixel in an intensity hologram, whereas complex numbers may be utilized in amplitude holograms and phase holograms. For the sake of simplicity, the intensity hologram is utilized here, but our algorithm may be readily employed also for complex-valued holograms. Let us commence by defining the notations in Table II based on Section III.

The coding rates $r_{0}, \ldots, r_{m-1}$ of Fig. 3 aim for maximizing the quality of the image reconstructed from the estimated hologram $\hat{U}$ at the receiver. However, as discussed in Section II, the reconstruction process involves multiple parameters, such as the wavelength of the laser, which makes the optimization of the reconstructed image at the transmitter a challenging task. In this paper, our objective is to maximize the peak signal-to-noise ratio PSNR of the estimated hologram $\hat{U}$, which represents the most popular objective video quality metric of the reconstructed image [42]. Defining the PSNR of the estimated hologram $\hat{U}$ as $\mathrm{PSNR}_{U}$, our objective function (OF) invoked for maximizing the quality of this hologram may be formulated as

$$
\underset{r_{0}, \ldots, r_{m-1}}{\arg } \max \left\{E\left(\mathrm{PSNR}_{U}\right)\right\}
$$

where the $\operatorname{PSNR}_{U}$ of the reconstructed hologram $\hat{U}$ may be calculated as

$$
\begin{aligned}
\operatorname{PSNR}_{U} & =10 \cdot \log _{10}\left\{\frac{\left(2^{m}-1\right)^{2}}{\mathrm{MSE}}\right\} d B \\
\mathrm{MSE} & =\frac{1}{W \cdot H} \sum_{w=0}^{H-1} \sum_{h=0}^{W-1}[U(\rho)-\hat{U}(\rho)]^{2}
\end{aligned}
$$

where the mean square error (MSE) is calculated based on the original hologram $U$ and the reconstructed hologram $\hat{U}$.

We note that $M S E$ is inversely proportional to $\mathrm{PSNR}_{U}$. By assuming that all pixels of $U$ obey an identical distribution, our $\mathrm{OF}$ of (5) may be expressed as

$$
\underset{r_{0}, \ldots, r_{m-1}}{\arg } \min \left\{\sum_{w=0}^{H-1} \sum_{h=0}^{W-1} E[U(\rho)-\hat{U}(\rho)]^{2}\right\}
$$

subject to the overall coding rate constraint of

$$
\sum_{i=0}^{m-1} \frac{1}{r_{i}}=\frac{m}{R}
$$

The hologram $\hat{U}$ of (7) is reconstructed from the FECdecoded bitplanes $\hat{U}_{0}, \ldots, \hat{U}_{m-1}$. Hence the estimated hologram $\hat{U}$ is jointly determined by the transceivers and FEC codecs of Fig. 3, as well as by the related FEC coding rates $r_{0}, \ldots, r_{m-1}$. These components of Fig. 3 cannot be analytically characterized, especially when considering diverse transceivers and FEC codecs may be employed. In Section IV-A, we will first propose our solution for characterizing the "demodulation-FEC decoding" operations at the receiver of Fig. 3 with the assistance of a lookup table (LUT). Then, in Sections IV-B and C, the OF of (7) will be cast in form of a multidimensional optimization problem, which will determine the optimal FEC coding rates $r_{0}, \ldots, r_{m-1}$ of Fig. 3. Finally, Section IV-D discusses the complexity issues imposed by the proposed techniques.

\section{A. Lookup Table}

Again, the "demodulation-FEC decoding" operations 4 of Fig. 3 cannot be analytically characterized for diverse system configurations, such as different transceivers, FEC generator polynomials, decoding metrics, etc, [37]. In our analysis, we consider the specific scenario that the $m$ FEC codecs of Fig. 3 are identical for the sake of simplicity. We model the "demodulation-FEC decoding" operations as a function of both the channel SNR and the coding rate $r$, which generates a specific bit error rate (BER) at its output. The following LUT is created correspondingly:

\footnotetext{
${ }^{4}$ Arbitrary modulation schemes and noniterative FEC may be readily applied.
} 
1) $T(\operatorname{snr}, r)$ : The BER value of the decoded sequence after the "demodulation-FEC decoding" operations, where $r$ represents the coding rate of the FEC codec. For example, $T\left(\operatorname{snr}, r_{i}\right)$ returns the BER of the sequence $\hat{\bar{u}}_{i}$, namely that of the bitplane $\hat{u}_{i}$, when the FEC codec $i$ has the coding rate $r_{i}$. Since this LUT relies both on the snr and on $r$, it may be stored in a 3-D memory. The LUT's memory requirements will be detailed in Section IV-D.

\section{B. Derivation of the $O F$}

Based on the discussions above, for the holographic pixel $\rho=(w, h)$, we have the following expressions

1) The pixels $U(\rho)$ and $\hat{U}(\rho)$ may be readily formulated as

$$
\begin{aligned}
& U(\rho)=\sum_{i=0}^{m-1} 2^{i} u_{i}(\rho) \\
& \hat{U}(\rho)=\sum_{i=0}^{m-1} 2^{i} \hat{u}_{i}(\rho)
\end{aligned}
$$

2) The probability that the reconstructed bit $\hat{u}_{j}(\rho)$ is 1 may be expressed as $p\left[\hat{u}_{j}(\rho)=1\right]$. According to the definition of the BER LUT $T(\mathrm{snr}, r)$, the probability $p\left[\hat{u}_{j}(\rho)=1\right]$ consists of the probability $p\left[u_{j}(\rho)=1\right]$. $\left[1-T\left(\mathrm{snr}, r_{j}\right)\right]$ indicating that the correctly decoded bit $\hat{u}_{j}(\rho)$ is 1 and the probability $\left[1-p\left[u_{j}(\rho)=1\right]\right]$. $T\left(\operatorname{snr}, r_{j}\right)$ indicating that the reconstructed bit $\hat{u}_{j}(\rho)$ is erroneous. Overall, the probability $p\left[\hat{u}_{j}(\rho)=1\right]$ may be expressed as

$$
\begin{aligned}
p\left[\hat{u}_{j}(\rho)=1\right]= & {\left[1-p\left[u_{j}(\rho)=1\right]\right] \cdot T\left(\mathrm{snr}, r_{j}\right) } \\
& +p\left[u_{j}(\rho)=1\right] \cdot\left[1-T\left(\mathrm{snr}, r_{j}\right)\right] .
\end{aligned}
$$

For the holographic pixel $\rho=(w, h)$, the expectation $E[U(\rho)-\hat{U}(\rho)]^{2}$ of (7) may be expressed as

$$
\begin{aligned}
E[U(\rho)-\hat{U}(\rho)]^{2}= & E\left[U^{2}(\rho)\right]-2 E[U(\rho) \cdot \hat{U}(\rho)] \\
& +E\left[\hat{U}^{2}(\rho)\right] .
\end{aligned}
$$

The component $E\left[U^{2}(\rho)\right]$ of (11) may be further formulated as

$$
\begin{aligned}
E\left[U^{2}(\rho)\right] & =E\left[\sum_{i=0}^{m-1} 2^{i} u_{i}(\rho)\right]^{2} \\
& =\sum_{i=0}^{m-1} \sum_{j=0}^{m-1} 2^{i+j} \cdot E\left[u_{i}(\rho) \cdot u_{j}(\rho)\right] .
\end{aligned}
$$

Similarly, for the components $E\left[\hat{U}^{2}(\rho)\right]$ and $E[U(\rho) \cdot \hat{U}(\rho)]$ of (11) we arrive at

$$
\begin{gathered}
E\left[\hat{U}^{2}(\rho)\right]=E\left[\sum_{i=0}^{m-1} 2^{i} \hat{u}_{i}(\rho)\right]^{2}=\sum_{i=0}^{m-1} \sum_{j=0}^{m-1} 2^{i+j} \\
\cdot E\left[\hat{u}_{i}(\rho) \cdot \hat{u}_{j}(\rho)\right]
\end{gathered}
$$

$$
\begin{aligned}
E[U(\rho) \cdot \hat{U}(\rho)] & =E\left[\sum_{i=0}^{m-1} 2^{i} u_{i}(\rho) \cdot \sum_{j=0}^{m-1} 2^{j} \hat{u}_{j}(\rho)\right] \\
& =\sum_{i=0}^{m-1} \sum_{j=0}^{m-1} 2^{i+j} \cdot E\left[u_{i}(\rho) \cdot \hat{u}_{j}(\rho)\right] .
\end{aligned}
$$

By substituting (12)-(14) into (11), the expectation $E[U(\rho)-$ $\hat{U}(\rho)]^{2}$ of (7) may be reformulated as

$$
\begin{aligned}
E[U(\rho)-\hat{U}(\rho)]^{2}= & \sum_{i=0}^{m-1} \sum_{j=0}^{m-1} 2^{i+j}\left\{E\left[u_{i}(\rho) \cdot u_{j}(\rho)\right]-2 E\right. \\
& \left.\times\left[u_{i}(\rho) \cdot \hat{u}_{j}(\rho)\right]+E\left[\hat{u}_{i}(\rho) \cdot \hat{u}_{j}(\rho)\right]\right\} .
\end{aligned}
$$

Since we have $u_{i}(\rho) \in\{0,1\}$ and $u_{j}(\rho) \in\{0,1\}$, the expectation $E\left[u_{i}(\rho) \cdot u_{j}(\rho)\right]$ of (15) may be expressed as

$$
E\left[u_{i}(\rho) \cdot \hat{u}_{j}(\rho)\right]=p\left[u_{i}(\rho)=1\right] \cdot p\left[\hat{u}_{j}(\rho)=1\right] .
$$

For $i=j$ and $u_{i}(\rho)=1$, the probability $p\left[\hat{u}_{j}(\rho)=1\right]$ of (16) represents the likelihood of the bit $u_{i}(\rho)$ being correctly decoded, which is given by ${ }^{5}\left[1-T\left(\operatorname{snr}, r_{i}\right)\right]$. Hence, we arrive at

$$
\begin{aligned}
& E\left[u_{i}(\rho) \cdot \hat{u}_{j}(\rho)\right] \\
& = \begin{cases}p\left[u_{i}(\rho)=1\right] \cdot\left[1-T\left(\operatorname{snr}, r_{i}\right)\right], & i=j \\
p\left[u_{i}(\rho)=1\right] \cdot p\left[\hat{u}_{j}(\rho)=1\right], & i \neq j .\end{cases}
\end{aligned}
$$

Similarly, for the expectations $E\left[u_{i}(\rho) \cdot u_{j}(\rho)\right]$ and $E\left[\hat{u}_{i}(\rho)\right.$. $\left.\hat{u}_{j}(\rho)\right]$ of $(15)$, we have

$E\left[u_{i}(\rho) \cdot u_{j}(\rho)\right]= \begin{cases}p\left[u_{i}(\rho)=1\right], & i=j \\ p\left[u_{i}(\rho)=1\right] \cdot p\left[u_{j}(\rho)=1\right], & i \neq j\end{cases}$

$E\left[\hat{u}_{i}(\rho) \cdot \hat{u}_{j}(\rho)\right]= \begin{cases}p\left[\hat{u}_{i}(\rho)=1\right], & i=j \\ p\left[\hat{u}_{i}(\rho)=1\right] \cdot p\left[\hat{u}_{i}(\rho)=1\right], & i \neq j .\end{cases}$

By substituting (10), (17)-(19) into (15), the component $E[U(w, h)-\hat{U}(w, h)]^{2}$ in the OF of (7) may be expressed as

$$
\begin{gathered}
E[U(\rho)-\hat{U}(\rho)]^{2}=\sum_{i=0}^{m-1} \sum_{j \in[0, m)}^{j \neq i} 2^{i+j} \cdot\left\{p\left[u_{i}(\rho)=1\right]\right. \\
\cdot p\left[u_{j}(\rho)=1\right]-2 \cdot p\left[u_{i}(\rho)=1\right] \cdot p\left[\hat{u}_{j}(\rho)=1\right] \\
\left.+p\left[\hat{u}_{i}(\rho)=1\right]\right\}+\sum_{i=0}^{m-1} 2^{2 i} \cdot\left\{p\left[u_{i}(\rho)=1\right]-2\right. \\
\left.\cdot p\left[u_{i}(\rho)=1\right] \cdot\left[1-T\left(\operatorname{snr}, r_{i}\right)\right]+p\left[\hat{u}_{i}(\rho)=1\right]\right\}
\end{gathered}
$$

where $p\left[\hat{u}_{i}(\rho)=1\right]$ is formulated by $p\left[u_{j}(\rho)=1\right]$ and $T\left(\mathrm{snr}, r_{j}\right)$ is given in (10).

\footnotetext{
${ }^{5}$ Note that the bitplane $u_{i}$ is encoded by the FEC encoder $i$ of Fig. 3 using the coding rate $r_{i}$.
} 


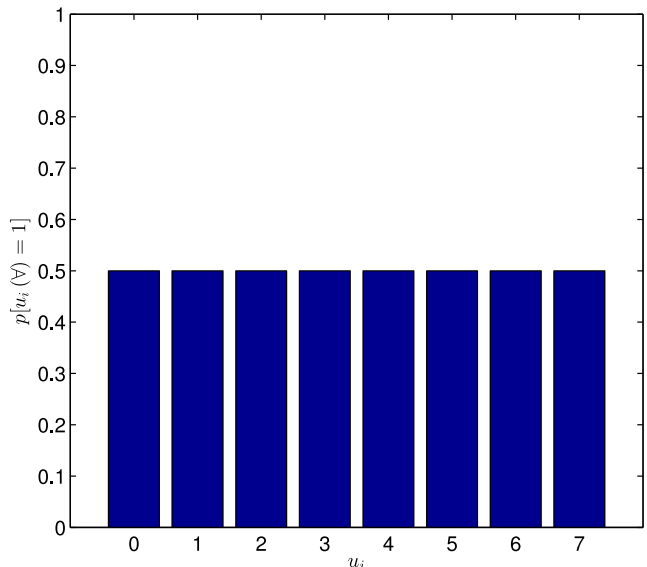

(a)

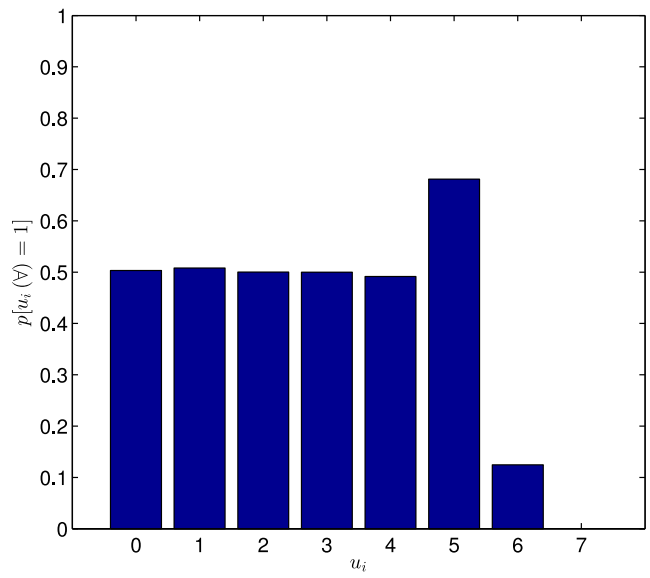

(b)

Fig. 4. Exemplified graph of $p\left[u_{i}(\forall)=1\right](0 \leq i<8)$. (a) Even distribution of bitplanes. (b) Bitplane distribution of the Coil hologram of [46].

\section{Optimal Rates}

Three components are involved in the expression of $E[U(\rho)-\hat{U}(\rho)]^{2}$ in (20), namely the snr, the coding rates $r_{0}, \ldots, r_{m-1}$ and the source distribution probability $p\left[u_{i}(\rho)=\right.$ $1]$, where $p\left[u_{i}(\rho)=1\right]$ may be readily obtained by scanning the source hologram $U$. We strike a tradeoff between the performance attained and the complexity imposed by assuming that all bits of the bitplane $u_{i}(0 \leq i<m)$ obey an identical distribution. Then, (20) is equivalent

$$
\begin{gathered}
E[U(\forall)-\hat{U}(\forall)]^{2}=\sum_{i=0}^{m-1} \sum_{j \in[0, m)}^{j \neq i} 2^{i+j} \cdot\left\{p\left[u_{i}(\forall)=1\right]\right. \\
\cdot p\left[u_{j}(\forall)=1\right]-2 \cdot p\left[u_{i}(\forall)=1\right] \cdot p\left[\hat{u}_{j}(\forall)=1\right] \\
\left.+p\left[\hat{u}_{i}(\forall)=1\right]\right\}+\sum_{i=0}^{m-1} 2^{2 i} \cdot\left\{p\left[u_{i}(\forall)=1\right]-2\right. \\
\left.\cdot p\left[u_{i}(\forall)=1\right] \cdot\left[1-T\left(\mathrm{snr}, r_{i}\right)\right]+p\left[\hat{u}_{i}(\forall)=1\right]\right\}
\end{gathered}
$$

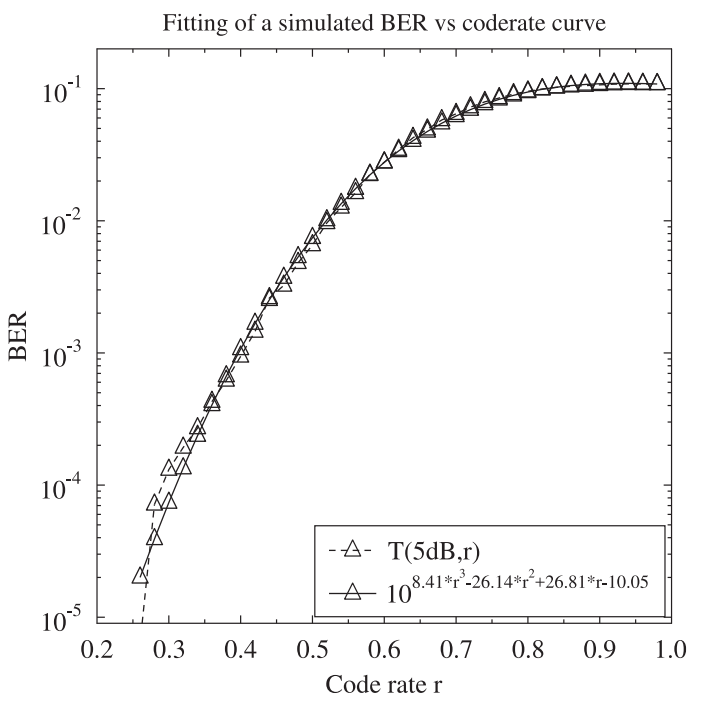

Fig. 5. Exemplified BER versus $r$ curve at $\mathrm{snr}=5 \mathrm{~dB}$ represented by LUT $T(5 \mathrm{~dB}, r)$ and the corresponding fitted curve of

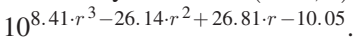

where $\forall$ indicates an arbitrary pixel-position in the bitplane $u_{i}$ and $p\left[\hat{u}_{i}(\forall)=1\right]$ is calculated as

$$
\begin{aligned}
p\left[\hat{u}_{i}(\forall)=1\right]= & {\left[1-p\left[u_{i}(\forall)=1\right]\right] \cdot T\left(\mathrm{snr}, r_{i}\right) } \\
& +p\left[u_{i}(\forall)=1\right] \cdot\left[1-T\left(\mathrm{snr}, r_{i}\right)\right] .
\end{aligned}
$$

Based on (21), the OF of (5) may be expressed as

$$
\underset{r_{0}, \ldots, r_{m-1}}{\arg } \min \left\{E[U(\forall)-\hat{U}(\forall)]^{2}\right\}
$$

subject to the overall coding rate constraint of

$$
\sum_{i=0}^{m-1} \frac{1}{r_{i}}=\frac{m}{R}
$$

Given a specific snr, the BER LUT $T\left(\mathrm{snr}, r_{i}\right)$ can be readily found by fitting a mathematical function. Finally, we may obtain the optimized coding rates $r_{0}, \ldots, r_{m-1}$ by solving the multidimensional optimization problem formulated in (23) under the condition of (24). ${ }^{6}$

The distribution of $p\left[u_{i}(\forall)=1\right](0 \leq i<m)$ is exemplified in Fig. 4. To elaborate a little further, we consider the example of Fig. 4(a), where we have $p\left[u_{i}(\forall)=1\right]=0.5(0 \leq i<m)$. Then the OF of (23) may be further simplified to

$$
\underset{r_{0}, \ldots, r_{m-1}}{\arg } \min \left\{\sum_{i=0}^{m-1} 4^{i} \cdot T\left(s n r, r_{i}\right)\right\} .
$$

\footnotetext{
${ }^{6}$ The Mathematica tool was employed in the simulations, while more solutions may be found in [43]-[45].
} 


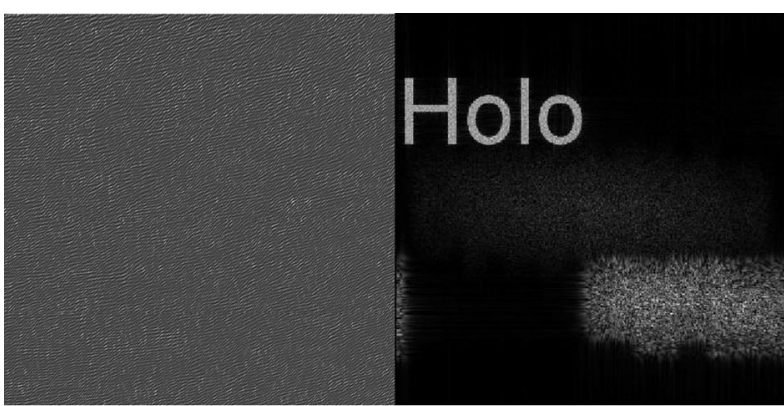

(a) (b)

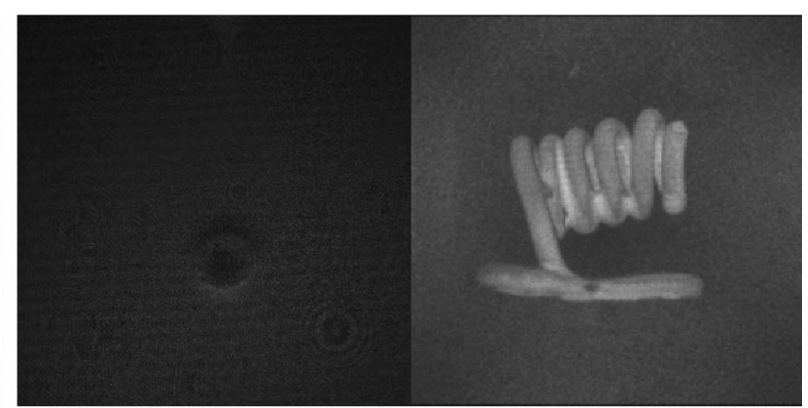

(c)

(d)

Fig. 6. Holo and Coil holograms employed. (a) Hologram of Holo. (b) Reconstructed image of the Holo Hologram. (c) Hologram of Coil. (d) Reconstructed image of the Coil Hologram.

TABLE III

THE PARAMETERS OF THE HOLOGRAMS EMPLOYED

\begin{tabular}{lcc}
\hline \hline & Holo & Coil \\
\hline Representation & YUV 4:0:0 & YUV 4:0:0 \\
Format & $256 \times 256$ & $2048 \times 2032$ \\
Bit-depth & 8 & 8 \\
Type & $\mathrm{CGH}$ & $\mathrm{DH}$ \\
Wavelength & $532 \mathrm{~nm}$ & $633 \mathrm{~nm}$ \\
Coding rates & $0.25 \sim 1$ & $0.25 \sim 1$ \\
Overall coding rate & $1 / 2$ & $1 / 2$ \\
\hline \hline
\end{tabular}

Moreover, we assume having $\mathrm{snr}=5 \mathrm{~dB}, R=\frac{1}{3}, 0.25 \leq$ $r_{i} \leq 1$, whereas the BER curve LUT $T(5 d B, r)$ of the 3 -D LUT plane at $\mathrm{snr}=5 \mathrm{~dB}$ is displayed in Fig. 5. The OF of (23) may be further simplified to

$$
\underset{r_{0}, \ldots, r_{m-1}}{\arg } \min \left\{\sum_{i=0}^{7} 4^{i} \cdot \exp \left(d+b r_{i}^{1}+b r_{i}^{2}+a r_{i}^{3}\right)\right\}
$$

subject to the constraint of

$$
\sum_{i=0}^{7} \frac{1}{r_{i}}=\frac{8}{1 / 3} .
$$

Finally, we obtain the optimal coding rates of $\left[r_{0}, \ldots, r_{7}\right]=$ $[1,1,1,0.53,0.45,0.39,0.34,0.30]$ by solving (26), resulting in a minimum MSE of $E(M S E)=E[U(\forall)-\hat{U}(\forall)]^{2}=6.67$ and a minimum of $\mathrm{PSNR}_{U}=39.9 \mathrm{~dB}$, respectively.

\section{Complexity Issues}

In the Opt-UEP-FEC scheme, the "coding rates optimization" block of Fig. 3 is the only part that imposes overheads compared to the typical EEP transmission scheme. These overheads include the generation of the LUT $T(\mathrm{snr}, r)$, the estimation of $p\left[u_{i}(\forall)=1\right](0 \leq i<m)$ and evaluating the $\mathrm{OF}$ of (23). Among these overheads, the generation of the LUT only imposes extra off-line design-time, whereas the estimation of $p\left[u_{i}(\forall)=1\right](0 \leq i<m)$ and the coding rate optimization impose extra on-line run-time complexity. Additionally, our system may be readily extended to complex-valued holograms, which approximately doubles the run-time complexity. Below, we analyze these complexity issues in order to characterize our system.
TABLE IV

EXAMPLE OF THE LUT $T$ (SNR, $r$ )

\begin{tabular}{lcc}
\hline \hline snr & $r$ & ber \\
\hline$\vdots$ & $\vdots$ & $\vdots$ \\
0 & 0.26 & 0.014 \\
0 & 0.28 & 0.035 \\
0 & 0.30 & 0.046 \\
$\vdots$ & $\vdots$ & $\vdots$ \\
0.5 & 0.26 & 0.008 \\
0.5 & 0.28 & 0.022 \\
0.5 & 0.30 & 0.030 \\
$\vdots$ & $\vdots$ & $\vdots$ \\
\hline \hline
\end{tabular}

1) Generation of LUT T (snr, $r)$ : The LUT $T(\mathrm{snr}, r)$ characterizes three components, namely the channel, the transceiver, and the FEC codec. Hence this LUT has to be regenerated, when any of these three components is changed. The LUT is independent of the holograms and it is generated during the design process. Furthermore, since the LUT is generated off-line, no extra run-time complexity is imposed by the LUT generation process for different channels, transceivers and FEC schemes. The size of this LUT depends on the number of snr and $r$ values. If $n_{\mathrm{snr}}$ and $n_{r}$ denote the number of snr and $r$ parameters, respectively, the LUT has a size of $\left(n_{\mathrm{snr}} \cdot n_{r}\right)$ entries. Furthermore, it costs constant time to generate each entry of the LUT. Hence the complexity depends on the size of the LUT. Overall, the generation of the LUT $T(\mathrm{snr}, r)$ imposes an off-line complexity of $O\left(n_{\mathrm{snr}} \cdot n_{r}\right)$ for time and space.

2) Estimation of $p\left[u_{i}(\forall)=1\right]$ : For a specific hologram, a one-off scanning is necessitated for estimating $p\left[u_{i}(\forall)=1\right]$, which represents a modest complexity. Moreover, the hologram $U$ has size of $(W \times H) m$-bit pixels. Hence the estimation of $p\left[u_{i}(\forall)=1\right]$ imposes a time complexity of $O(W \cdot H \cdot m)$ due to one time scanning of the hologram $U$.

3) Solving the OF: Again, solving the OF of (23) leads to a multidimensional optimization problem, which has been widely studied in the literature [43]-[45]. Specifically, the adaptive particle swarm optimization APSO technique of [45] may be readily employed for finding the global optimum in real-time. In our real-time simulations, we employed the Mathematica tool 


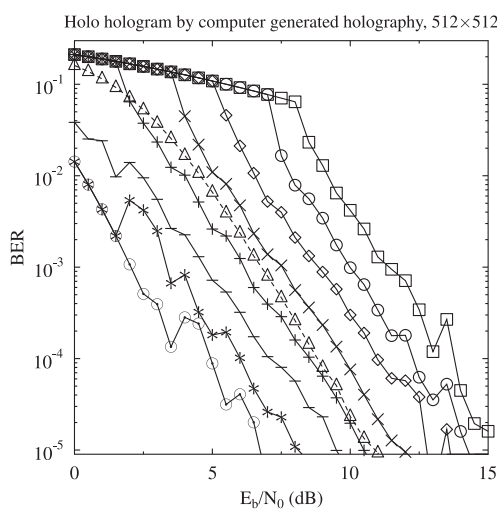

(a)

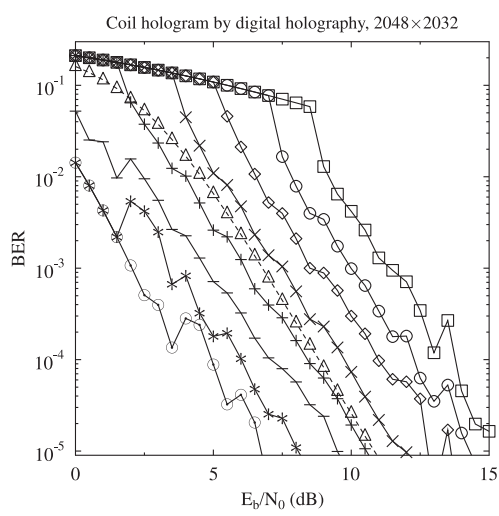

(d)

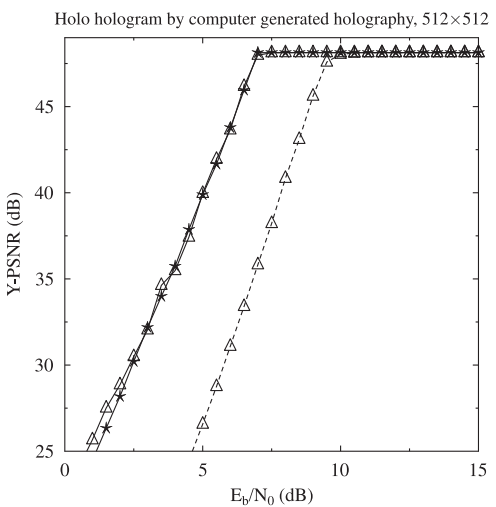

(b)

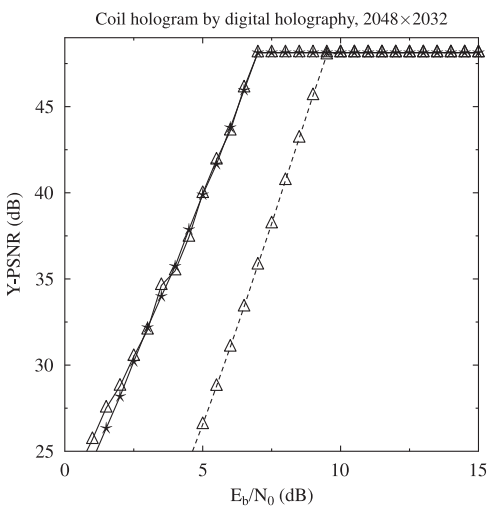

(e)

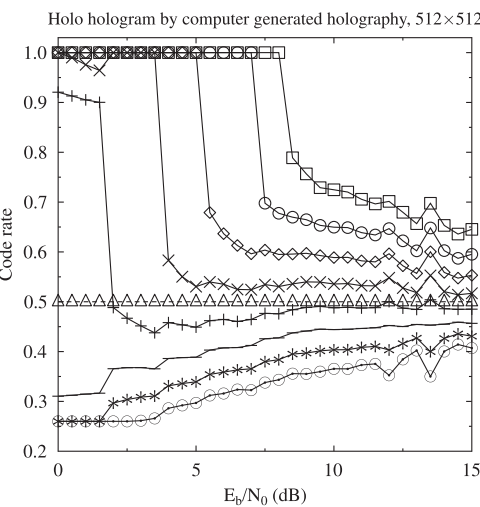

(c)

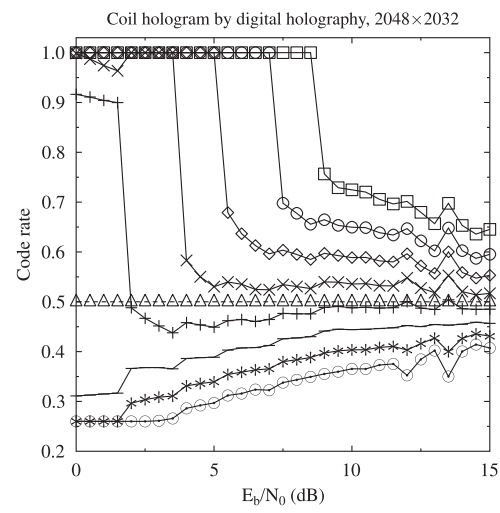

(f)

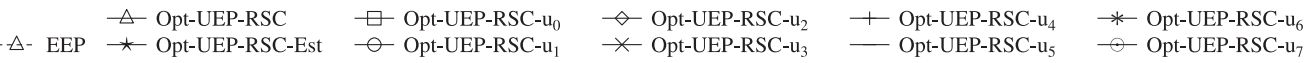

Fig. 7. BER, PSNR, Code rate versus $E_{b} / N_{0}$ performance comparison of the proposed system and the benchmarkers, namely the EEP-RSC scheme, the Opt-UEP-RSC scheme, and the Opt-UEP-RSC-Est scheme for the Holoand Coil holograms. (a) BER versus $E_{b} / N_{0}-H o l o$. (b) PSNR versus $E_{b} / N_{0}-H o l o$. (c) Code rate versus $E_{b} / N_{0}$ - Holo. (d) BER versus $E_{b} / N_{0}$ - Coil. (e) PSNR versus $E_{b} / N_{0}$ - Coil. (f) Code rate versus $E_{b} / N_{0}$ - Coil.

for obtaining the optimal coding rates $r_{0}, \ldots, r_{m-1}$. In conclusion, the complexity imposed by evaluating the OF depends on the specific multidimensional optimization solution employed.

4) Complex-Valued Holograms: For complex-valued holograms, we first split each complex pixel into its real and imaginary parts. Then, we apply our proposed techniques to the real and imaginary parts, respectively. Hence the complexity of evaluating the OF is doubled for complex-valued holograms compared to intensity holograms.

\section{SimUlations}

In this section, we benchmark our proposed OptUEP-RSC system against the traditional EEP-based FEC (EEP-FEC) system. Specifically, a $\mathrm{RSC}^{7}$ codec having the hexadecimally represented generator polynomials of $[1011,1101,1101,1111]$ is employed, resulting in the coding

\footnotetext{
${ }^{7}$ A recursive systematic convolutional (RSC) [46] code retains the original information bits in the encoded sequence and incorporates the parity bits. These parity bits are generated with the aid of a so-called recursive generator polynomial, which indicates that this encoder feeds back the parity bits for the computation of future parity bits. The benefits of this feedback is that the encoder has an infinite memory, which hence efficiently spreads the parity information over the encoded stream and, therefore, improves the decoding performance attained.
}

rate range of $[0.25,1]$. The overall coding rate of $1 / 2$ was employed. Moreover, BPSK modulated signals were transmitted through nondispersive uncorrelated Rayleigh fading wireless channels.

We employ the $m=8$ bit-depth intensity Holo and Coil holograms seen in Fig. 6, which are formatted in 4:0:0 YUV and represented in $(256 \times 256)$ - and $(2048 \times 2032)$-pixel formats, respectively. The Holo hologram was generated by CGH using a laser wavelength of $532 \mathrm{~nm}$ at a distance of $1.5 \mathrm{~m}$, whereas the Coil hologram [47] was digitally recorded using a laser wavelength of $633 \mathrm{~nm}$. The parameters of the holograms employed are listed in Table III. In all of our experiments, each hologram was transmitted 100 times in order to generate statistically sound performance curves.

\section{A. Off-Line LUT Generation}

In our experiments, the vectors of $[0: 0.5: 15]$ and $[0.26: 0.02: 1]^{8}$ are utilized for the variables snr and $r$, respectively, for generating the LUT, which result in $n_{\mathrm{snr}}=31$, $n_{r}=38$. For each snr value of $T(\mathrm{snr}, r)$, we recorded the BER achieved by the RSC decoder for the coding rates of

\footnotetext{
${ }^{8}$ These values can be stored as floats in 8 bytes each. The first and last element represent the interval limits, while the one in the middle is the step-size.
} 


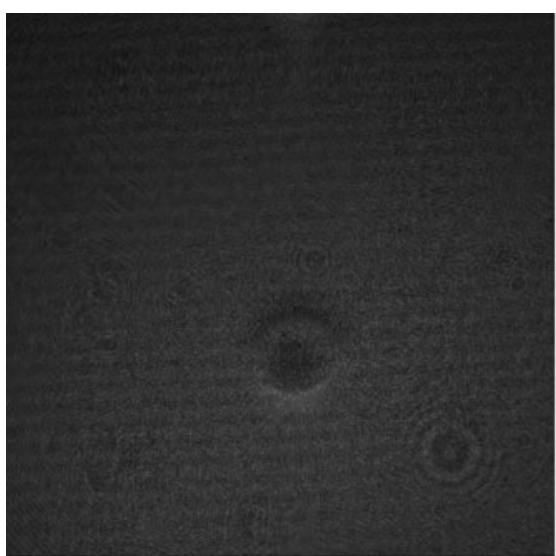

(a)

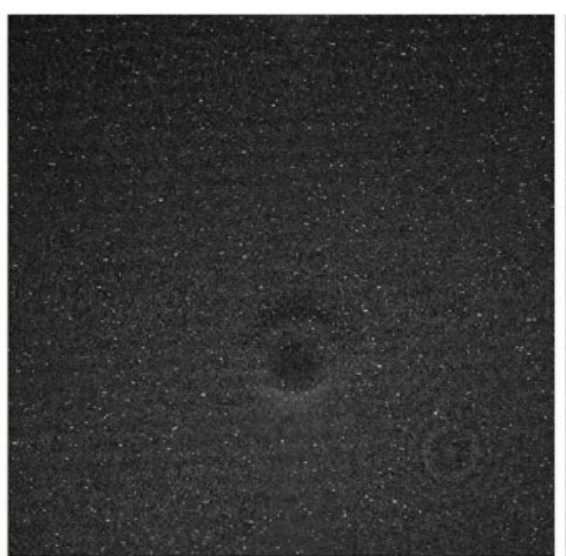

(b)

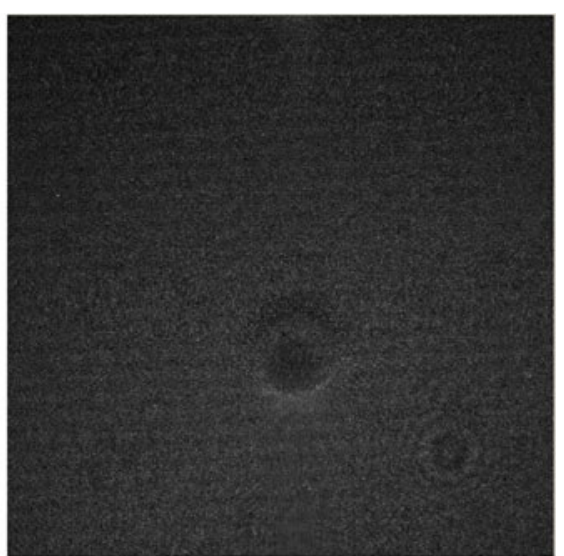

(c)
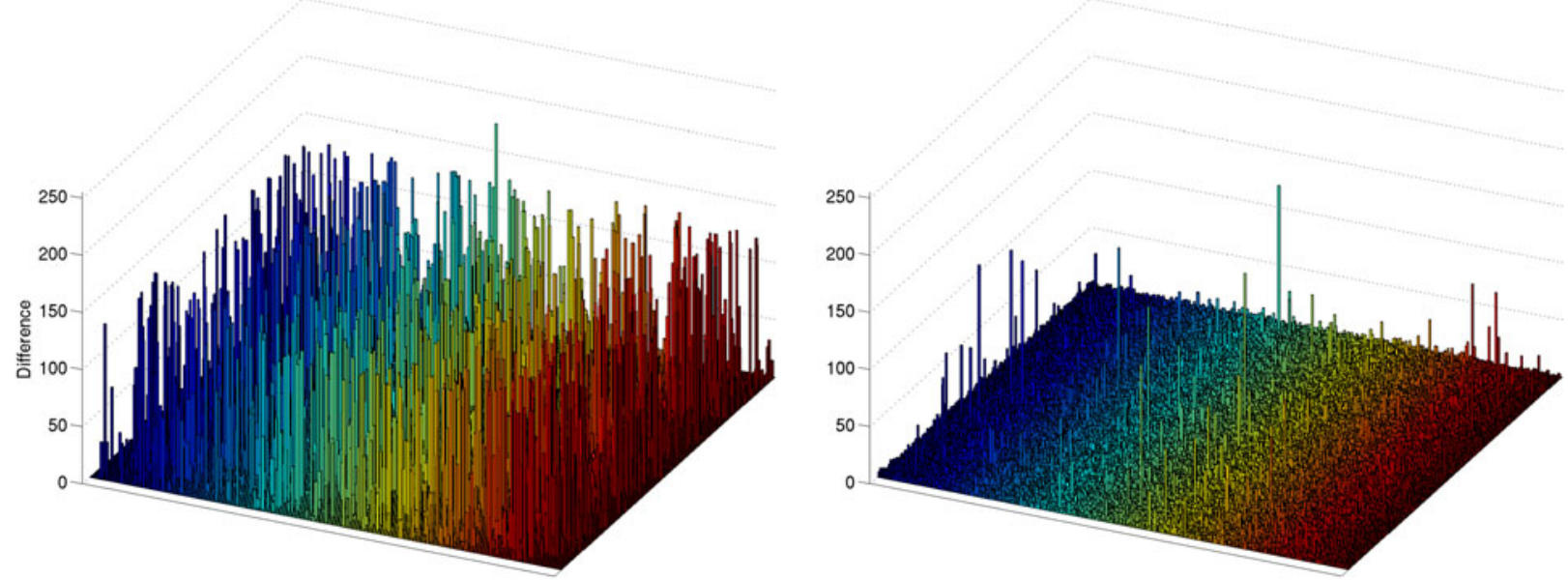

(d)

Fig. 8. Comparison of the frames at $E_{b} / N_{0}$ of $5 \mathrm{~dB}$ for the Coil hologram. (a) Original. (b) EEP-RSC. (c) Opt-UEP-RSC. (d) Comparison of difference between original and decoded holograms.

$[0.26: 0.02: 1]$. Furthermore, 8-byte floating values were utilized for storing the LUT in memory. Correspondingly, the LUT $T(\mathrm{snr}, r)$ requires memory sizes of about $\left(n_{\mathrm{snr}} \times n_{r}\right)=1178$ bytes. Some of the LUT entries generated for our system are displayed in Table IV.

\section{B. System Performance}

In this section, we benchmark our Opt-UEP-RSC system against the traditional EEP-RSC system. The BER versus $E_{b} / N_{0}$ curves of the eight bitplanes of the Holo hologram are displayed in Fig. 7(a). As expected, the BER of the bitplanes $u_{4}, \ldots, u_{7}$ of the Opt-UEP-RSC system is always better than that of the EEP-RSC system, while the BER of the bitplanes $u_{0}, \ldots, u_{3}$ is worse than that of the EEP-RSC system owing to the specific code rates. More specifically, this is due to the fact that the coding-rates of the bitplanes $u_{0}, \ldots, u_{3}$ are increased for the sake of protecting the more vunerable $u_{4}, \ldots, u_{7}$ bitplanes.
Similar trends were observed also for the Coil hologram, which are displayed in Fig. 7(d).

The PSNR versus $E_{b} / N_{0}$ performance recorded for the Holo hologram is displayed in Fig. 7(b), where the PSNR estimated using the techniques detailed in Section IV is also provided by the curve Opt-UEP-RSC-Est. We observe that the Opt-UEP-RSC scheme substantially outperforms the EEP-RSC system, whereas it has similar performance to the theoretical curve Opt-UEP-RSC-Est. Specifically, the OptUEP-RSC scheme achieves an $E_{b} / N_{0}$ reduction of about $2.6 \mathrm{~dB}$ compared to the EEP-RSC scheme at a PSNR of 48 dB. ${ }^{9}$ Alternatively, about $12.5 \mathrm{~dB}$ of PSNR hologram quality improvement is observed at an $E_{b} / N_{0}$ of $7 \mathrm{~dB}$. Furthermore, the PSNR versus $E_{b} / N_{0}$ performance of the Opt-UEP-RSC using the Coil hologram is portrayed in Fig. 7(e), where similar trends

\footnotetext{
${ }^{9} 48 \mathrm{~dB}$ represents that the signal is near losslessly received.
} 
to those of Fig. 7(b) were observed. A subjective comparison of the benchmarkers recorded for the Holo hologram is presented in Fig. 8. In the first row, the three columns (from left to right) indicate the original hologram as well as that of the EEP-RSC scheme and of the Opt-UEP-RSC scheme, respectively. In the second row, the first/second figure indicates the difference between the original and the EEP-RSC/Opt-UEP-RSC decoded hologram.

\section{Optimized Coding Rates}

The optimized coding rates found by our proposed regime for the Holo and Coil holograms are shown in Figs. 7(c) and (f), respectively. Specifically, the $y$-axis of Fig. 7(c) and (f) indicates the coding rates. Observe from Fig. 7(c) that the coding rates $r_{4}, \ldots, r_{7}$ found for the bitplanes $u_{4}, \ldots, u_{7}$ increase gradually as the $E_{b} / N_{0}$ increases, whereas opposite trends were observed for the coding rates $r_{0}, \ldots, r_{3}$. This is due to the fact the bitplanes $u_{0}, \ldots, u_{3}$ were protected less well for the sake of protecting the more important bitplanes $u_{4}, \ldots, u_{7}$ at lower $E_{b} / N_{0}$ values. At high $E_{b} / N_{0}$ values, more RSC protection bits were allocated to the less important bitplanes $u_{0}, \ldots, u_{3}$, since better channel conditions result in a lower BER of the bitplanes $u_{4}, \ldots, u_{7}$, which freed up part of the RSC protection bits reassigned from the bitplanes $u_{0}, \ldots, u_{3}$. Similar trends may be observed for the Coil hologram, as displayed in Fig. 7(f).

\section{CONCLUSION}

We proposed a UEP-FEC technique for the bitplane-based transmission of digital holograms over wireless channels, where the coding rates of different bitplanes were optimized for the sake of achieving an improved hologram quality. First, the transceiver and soft-decoded FEC are treated as a black box, which was modeled by a LUT. Then, the PSNR of the hologram decoded at the receiver was expressed as a function of FEC coding rates of the $m$ independently encoded bitplanes. Finally, we solved the resultant multidimensional optimization problem of generating the optimal coding rates for the $m$ bitplanes. Numerical simulation of a pair of holograms were provided, which shows that the proposed Opt-UEP-FEC system outperforms the traditional UEP-FEC system by up to 2.6 $\mathrm{dB}$ of $E_{b} / N_{0}$ or $12.5 \mathrm{~dB}$ of PSNR, when employing an RSC code.

In our future work, we may incorporate our previous interlayer FEC technique [37], [38] into our digital hologram transmission system. Moreover, we may consider compressing the digital holograms using lossless variable length coding (VLC) [48], [49], which is capable of soft decoding.

\section{REFERENCES}

[1] D. Gabor, "A new microscopic principle," Nature, vol. 161, no. 4098, pp. 777-778, May 1948.

[2] M. Kujawinska et al., "Multiwavefront digital holographic television," Opt. Exp., vol. 22, no. 3, pp. 2324-2336, Feb. 2014. [Online]. Available: http://oe.osa.org/abstract.cfm?URI=oe-22-3-2324
[3] M. Lin, K. Nitta, O. Matoba, and Y. Awatsuji, "Parallel phase-shifting digital holography with adaptive function using phase-mode spatial light modulator," Appl. Opt., vol. 51, no. 14, pp. 2633-2637, May 2012. [Online]. Available: http://ao.osa.org/abstract.cfm?URI=ao-51-14-2633

[4] Y. Ohsawa, K. Yamaguchi, T. Ichikawa, and Y. Sakamoto, "Computergenerated holograms using multiview images captured by a small number of sparsely arranged cameras," Appl. Opt., vol. 52, no. 1, pp. A167-A176, Jan. 2013. [Online]. Available: http://ao.osa.org/abstract.cfm?URI=ao52-1-A167

[5] L. Huang et al., "Three-dimensional optical holography using a plasmonic metasurface," Nature Commun., vol. 4, Nov. 2013, Art. no. 2808. [Online]. Available: http://dx.doi.org/10.1038/ncomms3808

[6] B. J. Jackin and T. Yatagai, "Fast calculation of spherical computer generated hologram using spherical wave spectrum method," Opt. Express, vol. 21, no. 1, pp. 935-948, Jan. 2013. [Online]. Available: http://www.opticsexpress.org/abstract.cfm?URI=oe-21-1-935

[7] L. Onural, F. Yaras, and H. Kang, "Digital holographic threedimensional video displays," Proc. IEEE, vol. 99, no. 4, pp. 576-589, Apr. 2011.

[8] Y. N. Denisyuk, "Photographic reconstruction of the optical properties of an object in its own scattered radiation field," Soviet Phys. Doklady, vol. 7, pp. $543-545,1962$.

[9] E. N. Leith and J. Upatnieks, "Wavefront reconstruction with diffused illumination and three-dimensional objects," J. Opt. Soc. Amer. $B$, vol. 54, no. 11, pp. 1295-1301, Nov. 1964. [Online]. Available: http://dx.doi.org/10.1364/josa.54.001295

[10] B. R. Brown and A. W. Lohmann, "Complex spatial filtering with binary masks," Appl. Opt., vol. 5, no. 6, pp. 967-969, Jun. 1966. [Online]. Available: http://ao.osa.org/abstract.cfm?URI=ao-5-6-967

[11] A. W. Lohmann and D. P. Paris, "Binary Fraunhofer holograms, generated by computer," Appl. Opt., vol. 6, no. 10, pp. 1739-48, Oct. 1967. [Online]. Available: http://www.biomedsearch.com/nih/Binary-fraunhoferholograms-generated-b y/20062296.html

[12] J. W. Goodman and R. W. Lawrence, "Digital image formation from electronically detected holograms," Appl. Phys. Lett., vol. 11, pp. 77-79, 1967.

[13] M. K. Kim, "Principles and techniques of digital holographic microscopy," J. Photon. Energy, vol. 1, pp. 018005-1-018005-50, 2010. [Online]. Available: http://dx.doi.org/10.1117/6.0000006

[14] L. Yaroslavskii and N. Merzlyakov, Methods of Digital Holography. New York, NY, USA: Consultants Bureau, 1980.

[15] U. Schnars and W. Jüptner, Digital Holography: Digital Hologram Recording, Numerical Reconstruction, and Related Techniques. New York, NY, USA: Springer, 2005. [Online]. Available: http://books.google. co.uk/books?id $=$ oJEr304N7isC

[16] I. Moon, M. Daneshpanah, B. Javidi, and A. Stern, "Automated threedimensional identification and tracking of micro/nanobiological organisms by computational holographic microscopy," Proc. IEEE, vol. 97, no. 6, pp. 990-1010, Jun. 2009.

[17] G. Pedrini, M. Gusev, S. Schedin, and H. Tiziani, "Pulsed digital holographic interferometry by using a flexible fiber endoscope," Opt. Lasers Eng., vol. 40, no. 5/6, pp. 487-499, Nov. 2003. [Online]. Available: http://www.sciencedirect.com/science/article/pii/S0143816602000775

[18] R. Gazdzinski and L. Rosen, "Error-corrected wideband holographic communications apparatus and methods," U.S. Patent Application 10/867,794, May 2005. [Online]. Available: http://www.google.com/ patents/US20050100102

[19] Y. Huo, C. Hellge, T. Wiegand, and L. Hanzo, "A tutorial and review on inter-layer FEC coded layered video streaming," IEEE Commun. Surveys Tut., vol. 17, no. 2, pp. 1166-1207, 2nd Quar. 2015.

[20] Y. Frauel, T. Naughton, O. Matoba, E. Tajahuerce, and B. Javidi, "Three-dimensional imaging and processing using computational holographic imaging," Proc. IEEE, vol. 94, no. 3, pp. 636-653, Mar. 2006.

[21] L. Onural, A. Gotchev, H. Ozaktas, and E. Stoykova, "A survey of signal processing problems and tools in holographic three-dimensional television," IEEE Trans. Circuits Syst. Video Technol., vol. 17, no. 11, pp. 1631-1646, Nov. 2007.

[22] T. J. Naughton, J. B. McDonald, and B. Javidi, "Efficient compression of fresnel fields for internet transmission of three-dimensional images," Appl. Opt., vol. 42, no. 23, pp. 4758-4764, Aug. 2003. [Online]. Available: http://ao.osa.org/abstract.cfm?URI=ao-42-23-4758

[23] M. Liebling, T. Blu, and M. Unser, "Fresnelets: New multiresolution wavelet bases for digital holography," IEEE Trans. Image Process., vol. 12, no. 1, pp. 29-43, Jan. 2003. 
[24] E. Darakis and J. Soraghan, "Use of fresnelets for phase-shifting digital hologram compression," IEEE Trans. Image Process., vol. 15, no. 12, pp. 3804-3811, Dec. 2006.

[25] A. Shortt, T. J. Naughton, and B. Javidi, "Compression of digital holograms of three-dimensional objects using wavelets," Opt. Exp., vol. 14, no. 7, pp. 2625-2630, Apr. 2006. [Online]. Available: http://www.opticsexpress.org/abstract.cfm?URI=oe-14-7-2625

[26] L. T. Bang, Z. Ali, P. D. Quang, J.-H. Park, and N. Kim, "Compression of digital hologram for three-dimensional object using waveletbandelets transform," Opt. Exp., vol. 19, no. 9, pp. 8019-8031, Apr. 2011. [Online]. Available: http://www.opticsexpress.org/abstract. cfm?URI=oe-19-9-8019

[27] H. Schwarz, D. Marpe, and T. Wiegand, "Overview of the scalable video coding extension of the H.264/AVC standard," IEEE Trans. Circuits Syst. Video Technol., vol. 17, no. 9, pp. 1103-1120, Sep. 2007

[28] Y.-H. Seo, Y.-H. Lee, J.-S. Yoo, and D.-W. Kim, "Scalable hologram video coding for adaptive transmitting service," Appl. Opt., vol. 52, no. 1, pp. A254-A268, Jan. 2013. [Online]. Available: http://ao.osa.org/ abstract.cfm?URI=ao-52-1-A254

[29] K. Takano, K. Sato, T. Endo, H. Asano, A. Fukuzawa, and K. Asai, “An elementary research on wireless transmission of holographic 3D moving pictures," Proc. SPIE, vol. 7358, pp. 73 581A-1-73 581A-11, May 2009. [Online]. Available: http://dx.doi.org/10.1117/12.820575

[30] R. D. Leonardo and S. Bianchi, "Hologram transmission through multimode optical fibers," Opt. Exp., vol. 19, no. 1, pp. 247-254, Jan. 2011. [Online]. Available: http://www.opticsexpress.org/abstract.cfm?URI=oe19-1-247

[31] K. Sato, M. Tozuka, K. Takano, and M. Ohki, "Transmission of hologram data and 3D image reconstruction using white LED light," Proc. SPIE, vol. 8281, 2012, Art. no. 82810A. [Online]. Available: http://dx.doi.org/10.1117/12.907246

[32] M. Tozuka, K. Takano, K. Sato, and M. Ohki, "Digital space transmission of an interference fringe-type computer-generated hologram using IrSimple," in Proc. ITU Kaleidoscope, Building Sustain. Communities, Apr. 2013, pp. 1-6.

[33] Y. Huo, C. Zhu, and L. Hanzo, "Spatio-temporal iterative source-channel decoding aided video transmission," IEEE Trans. Veh. Technol., vol. 62, no. 4, pp. 1597-1609, May 2013.

[34] Y. Huo, T. Wang, R. G. Maunder, and L. Hanzo, "Iterative source and channel decoding relying on correlation modelling for wireless video transmission," IET Commun., vol. 7, pp. 1465-1475, Sep. 2013. [Online]. Available: http://digital-library.theiet.org/content/journals/ 10.1049/iet-com.2013.0053

[35] Joint Photographic Experts Group ISO/IEC, JTC/SC/WG8, CCITT SGVIII, "JPEG technical specifications, revision 5," Int. Telecommun. Union, Geneva, Switzerland, Rep. JPEG-8-R5, Jan. 1990.

[36] AVC: Advanced Video Coding for Generic Audiovisual Services, Joint Video Team (JVT) of ISO/IEC MPEG and ITU-T VCEG, ITU-T Rec. H.264/ISO/IEC 14496-10, Mar. 2010.

[37] Y. Huo, M. El-Hajjar, R. G. Maunder, and L. Hanzo, "Layered wireless video relying on minimum-distortion inter-layer FEC coding," IEEE Trans. Multimedia, vol. 23, no. 1, pp. 319-331, Jan. 2014.

[38] Y. Huo, M. El-Hajjar, and L. Hanzo, "Inter-layer FEC aided unequal error protection for multilayer video transmission in mobile TV," IEEE Trans. Circuits Syst. Video Technol., vol. 23, no. 9, pp. 1622-1634, Sep. 2013.

[39] Y. Huo, C. Hellge, T. Wiegand, and L. Hanzo, "A tutorial and review on inter-layer FEC coded layered video streaming," IEEE Commun. Surveys Tut., vol. 17, no. 2, pp. 1166-1207, 2nd Quar., 2015.

[40] T. Poon, Digital Holography and Three-Dimensional Display: Principles and Applications. New York, NY, USA: Springer, 2006.

[41] P. Picart and J. Li, Digital Holography (Series ISTE). Hoboken, NJ, USA: Wiley, 2013. [Online]. Available: http://books.google.co.uk/ Books?id= whzxBiOSiboC

[42] Q. Huynh-Thu and M. Ghanbari, "Scope of validity of PSNR in image/video quality assessment," Electron. Lett., vol. 44, no. 13, pp. 800-801, Jun. 2008.

[43] M. Clerc and J. Kennedy, "The particle swarm-explosion, stability, and convergence in a multidimensional complex space," IEEE Trans. Evol. Comput., vol. 6, no. 1, pp. 58-73, Feb. 2002.

[44] D. Schonfeld and N. Bouaynaya, "A new method for multidimensional optimization and its application in image and video processing," IEEE Signal Process. Lett., vol. 13, no. 8, pp. 485-488, Aug. 2006.
[45] Z.-H. Zhan, J. Zhang, Y. Li, and H.-H. Chung, "Adaptive particle swarm optimization,” IEEE Trans. Syst. Man, Cybern. B, Cybern., vol. 39, no. 6, pp. 1362-1381, Dec. 2009.

[46] C. Berrou and A. Glavieux, "Near optimum error correcting coding and decoding: Turbo codes," IEEE Trans. Commun., vol. 44, no. 10 pp. 1261-1271, Oct. 1996.

[47] E. Darakis, M. Kowiel, R. Näsänen, and T. J. Naughton, "Visually lossless compression of digital hologram sequences," Proc. SPIE, vol. 7529, 2010 Art. no. 752912.

[48] L. Hanzo, P. Cherriman, and J. Streit, Video Compression and Communications: From Basics to H.261, H.263, H.264, MPEG2, MPEG4 for DVB and HSDPA-Style Adaptive Turbo-Transceivers. New York, NY, USA: Wiley, 2007.

[49] L. Hanzo, R. G. Maunder, J. Wang, and L.-L. Yang, Near-Capacity Variable-Length Coding: Regular and Exit-Chart Aided Irregular Designs. New York, NY, USA: Wiley, 2010.

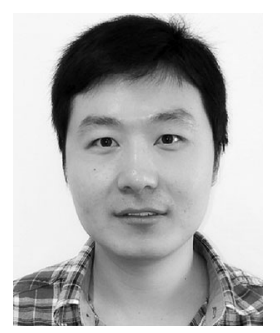

Yongkai Huo received the B.Eng. degree with distinction in computer science and technology from Hefei University of Technology, Hefei, China, in 2006; the M.Eng. degree in computer software and theory from University of Science and Technology of China, Hefei, in 2009; and the Ph.D. degree from the Wireless Communications Group, School of Electronics and Computer Science, University of Southampton, Southampton, U.K., in 2014.

He was a Research Fellow until September 2016 with the University of Southampton. He is currently an Associate Professor with the College of Computer Science and Software Engineering, Shenzhen University, Shenzhen, China. He has published a number of research papers on holographic images, distributed video coding, multiview video coding, robust wireless video streaming and joint source-channel decoding, etc.

Dr. Huo received a scholarship under the China-U.K. Scholarships for Excellence Programme.

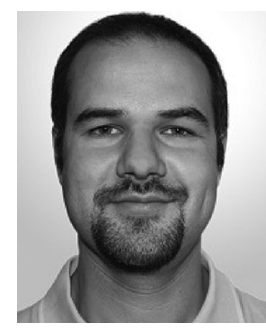

Péter Tamás Kovács (S'14) received the M.Sc. degree in computer science from the Budapest University of Technology, Budapest, Hungary, in 2004. He is currently working toward the Ph.D. degree in signal processing with Tampere University of Technology, Tampere, Finland.

From 2004 to 2006, he was a Software Engineer with Archi-Data. Since 2006, he has been a Software Engineer, Lead Software Engineer, then CTO of Holografika. He was a Visiting Researcher with Tampere University of Technology from 2013 to 2014 . He is the author or coauthor of three book chapters, three journal papers, and more than 30 conference papers. His research interests include 3-D displays, more specifically light-field displays, and the capture/compression/rendering of light fields.

Dr. Kovács has been a Program Committee member for numerous IEEE International Conferences, Local Organizing Chair of 3-DTV-Con 2014, and is a contributing member of the International 3-D Society, as well as the International Committee for Display Metrology, where he contributed to the first IDMS standard. He was the Head of Delegation to MPEG for Hungary. He was also the Chair of the Working Group 5 ("3-D End-User Devices") of the 3-D-ConTourNet COST Action. 


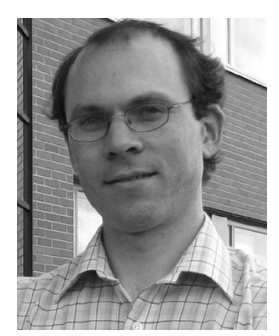

Thomas J. Naughton received the B.Sc. (Double Hons.) degree in computer science and experimental physics from Maynooth University (formerly St. Patrick's College) in 1995.

$\mathrm{He}$ is currently a Professor with the Maynooth University Department of Computer Science. His research and teaching areas are related to digital holography, optical image processing, and computer theory. He has published over 200 peer-reviewed publications and supervised six Ph.D. students to completion. During 2008-2011, he was the Coordinator and Scientific Leader of the 40-person-year European Commission FP7 Collaborative Project about digital holography called "Real 3D.” During 2007-2009, he was a European Commission Marie Curie Fellow based at the University of Oulu, Finland.

Prof. Naughton was jointly awarded the IEEE Donald G. Fink Prize Paper Award in 2008.

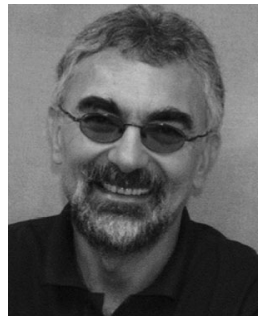

Lajos Hanzo (M'90-SM'91-F'04) received the M.S degree in electronics and the Ph.D. degree from the Technical University of Budapest, Budapest, Hungary, in 1976 and 1983, respectively.

He received the prestigious Doctor of Sciences Research degree in wireless communications from the University of Southampton, Southampton, U.K., in 2004. In 2016, he joined the Hungarian Academy of Science, Budapest, Hungary. During his 40-year career in telecommunications, he held various research and academic posts in Hungary, Germany, and the U.K. Since 1986, he has been with the School of Electronics and Computer Science, University of Southampton, U.K., where he holds the Chair in telecommunications. He has successfully supervised $111 \mathrm{Ph}$.D. students, coauthored 20 John Wiley/IEEE Press books on mobile radio communications, totaling in excess of 10000 pages, published 1600+ research contributions on IEEE Xplore, acted both as a Technical Program Committee member and the General Chair of IEEE conferences, presented keynote lectures, and received a number of distinctions. Currently he is directing a 60-strong academic research team, working on a range of research projects in the field of wireless multimedia communications sponsored by industry, The Engineering and Physical Sciences Research Council, U.K., and The European Research Council's Advanced Fellow Grant. $\mathrm{He}$ is an enthusiastic supporter of industrial and academic liaison, and he offers a range of industrial courses. He has $25000+$ citations and an H-index of 60 . For further information on research in progress and associated publications, see http://www-mobile.ecs.soton.ac.uk.

Dr. Hanzo is a Governor of the IEEE Vehicular TeChNOLOGy Society. During 2008-2012, he was the Editor-in-Chief of the IEEE Press and a Chaired Professor with Tsinghua University, Beijing, China. In 2009, he received an honorary doctorate Award by the Technical University of Budapest and in 2015, from the University of Edinburgh, Edinburgh, U.K., as well as the Royal Society's Wolfson Research Merit Award. He is a Fellow of the Royal Academy of Engineering, The Institution of Engineering and Technology, and EURASIP. 